\title{
Evaluation of find
}

\section{your talent -}

\section{overview report}

\section{SQW Consulting}

Department for 
This research report was commissioned before the new UK Government took office on 11 May 2010. As a result the content may not reflect current Government policy and may make reference to the Department for Children, Schools and Families (DCSF) which has now been replaced by the Department for Education (DFE).

The views expressed in this report are the authors' and do not necessarily reflect those of the Department for Education. 


\section{Contents}

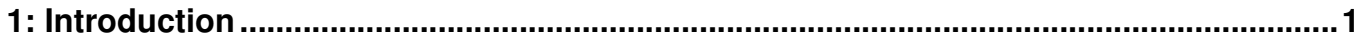

2: Local area characteristics and participation ...............................................................5

3: Area-based delivery models .................................................................................... 11

4: Pathfinder effectiveness ......................................................................................... 16

5: Conclusion - making a difference ........................................................................2 27

\begin{tabular}{lllll}
\hline Contact: & Michael Frearson & Tel: & $01223209400 \quad$ email: mfrearson@sqw.co.uk \\
\hline & & & \\
\hline Approved by: & Michael Frearson & Date: & 25 October 2010 & \\
& Associate Director & & & \\
& & &
\end{tabular}




\section{1: Introduction}

1.1 This report provides an overview of the main findings of the Find Your Talent (FYT) programme evaluation. Following the creation of the coalition government in May 2010, it was announced by HM Treasury on $17^{\text {th }}$ June that a decision to cancel FYT had been taken as part of a review of pilot programmes established by the previous government. The evaluation of the programme was also terminated as a result of this announcement. This report therefore presents evaluation findings to date in order to capture lessons learned.

\section{Find Your Talent}

1.2 The FYT programme commenced with an announcement of a pilot of a cultural offer to all children and young people in England in The Children's Plan (DCSF, December 2007) comprising an entitlement to five hours per week of high-quality cultural experiences. The intention was to help ensure children and young people stay on the path to success by participating in positive activities and experiences which develop their talents.

1.3 The programme aimed to offer high quality cultural experiences to all children and young people through a more co-ordinated and coherent offer and better local area planning of provision. The government committed to spend $£ 25 \mathrm{~m}$ from 2008 to 2011 through ten pathfinder areas where local authorities and cultural and other partners would trial different ways of delivering the cultural offer. In May 2008 the ten pathfinders were selected from 141 expressions of interest and the programme was launched in September 2008. Creativity, Culture and Education (CCE) was commissioned to manage the delivery of the programme, overseen by the Department of Culture, Media and Sport (DCMS) and the Department for Education (DfE) and a steering group which included representatives from the Arts Council for England and the Museums, Libraries and Archives Council (MLA). ${ }^{2}$

1.4 Existing evidence suggests that engagement in cultural activities can be beneficial to a young person's development by enhancing transferable and inter-personal skills and improving motivation and behaviour. Potentially, greater take up of cultural activities could enhance and extend learning opportunities and help improve outcomes for children, as outlined in the Every Child Matters framework ${ }^{3}$. The evaluation set out to test this hypothesis across a range of different FYT delivery models and local area characteristics.

1.5 There are clear market failures to cultural provision and participation for children and young people. The current cultural offer is fragmented and poorly co-ordinated, making it hard for users to gain access, especially those that are particularly hard to reach. Hence the rationale for intervention lay in extending the reach and range of the current cultural offer, and in

\footnotetext{
${ }^{1}$ Note that as a result of the formation of a new government in May 2010, the programme was subsequently terminated in June 2010 and no longer exists.

${ }^{2}$ Management and governance of the FYT programme were not included in the terms of reference for the evaluation.

http://publications.everychildmatters.gov.uk/default.aspx?PageFunction=productdetails\&PageMode=publications \&ProductId=DCSF-00331-2008
} 
providing high quality and better co-ordinated cultural experiences for children and young people.

1.6 The programme attempted to do this by strengthening in-school and out-of-school provision in local areas in order to widen participation for those accessing low or no levels of cultural activity. The aim was to remove barriers to access and increase choice, opportunity and the quality of the cultural offer for the entire 0-19 population in the ten pilot areas. The programme also aimed to support local and regional economic strategies by nurturing talent and encouraging progression into employment in the creative and cultural industries.

\section{The evaluation}

1.7 In October 2008, SQW, together with Ipsos MORI and Wafer Hadley, were commissioned by the (then) DCSF and DCMS to evaluate the FYT programme in the ten pathfinder areas ${ }^{4}$. The overall purpose was to investigate:

What works best in the delivery of high-quality cultural experiences for children and young people and determine what is needed to deliver this offer to all children aged $0-19$ in different localities. ${ }^{5}$

1.8 The aim of the evaluation was to assess the impact of the programme in the ten pathfinder areas between 2008 and 2011, and to construct the evidence base for future investment based on different starting points for different delivery models in different places. Specifically, the evaluation was designed to test whether, and the extent to which:

- the programme brings about increases in participation and take up of the cultural offer, especially among the hard-to-reach

- there are improvements in the overall quality, co-ordination and coherence of the offer

- the offer delivers cultural, social and other wider outcomes.

1.9 Our approach to the evaluation was designed to provide a robust and systematic evidence across five core themes:

- $\quad$ participation

- outcomes

- $\quad$ quality

- $\quad$ delivery models

- $\quad$ value for money.

1.10 Figure 1-1 outlines the evaluation work programme for data collection, analysis and reporting.

\footnotetext{
${ }^{4}$ Tower Hamlets, Leeds, Liverpool, Customs House (North and South Tyneside), Bolton, PUSH (South Hampshire), Shepway, Telford, North Somerset and Leicester and Leicestershire.

${ }^{5}$ DCSF/DCMS Evaluation Terms of Reference.
} 


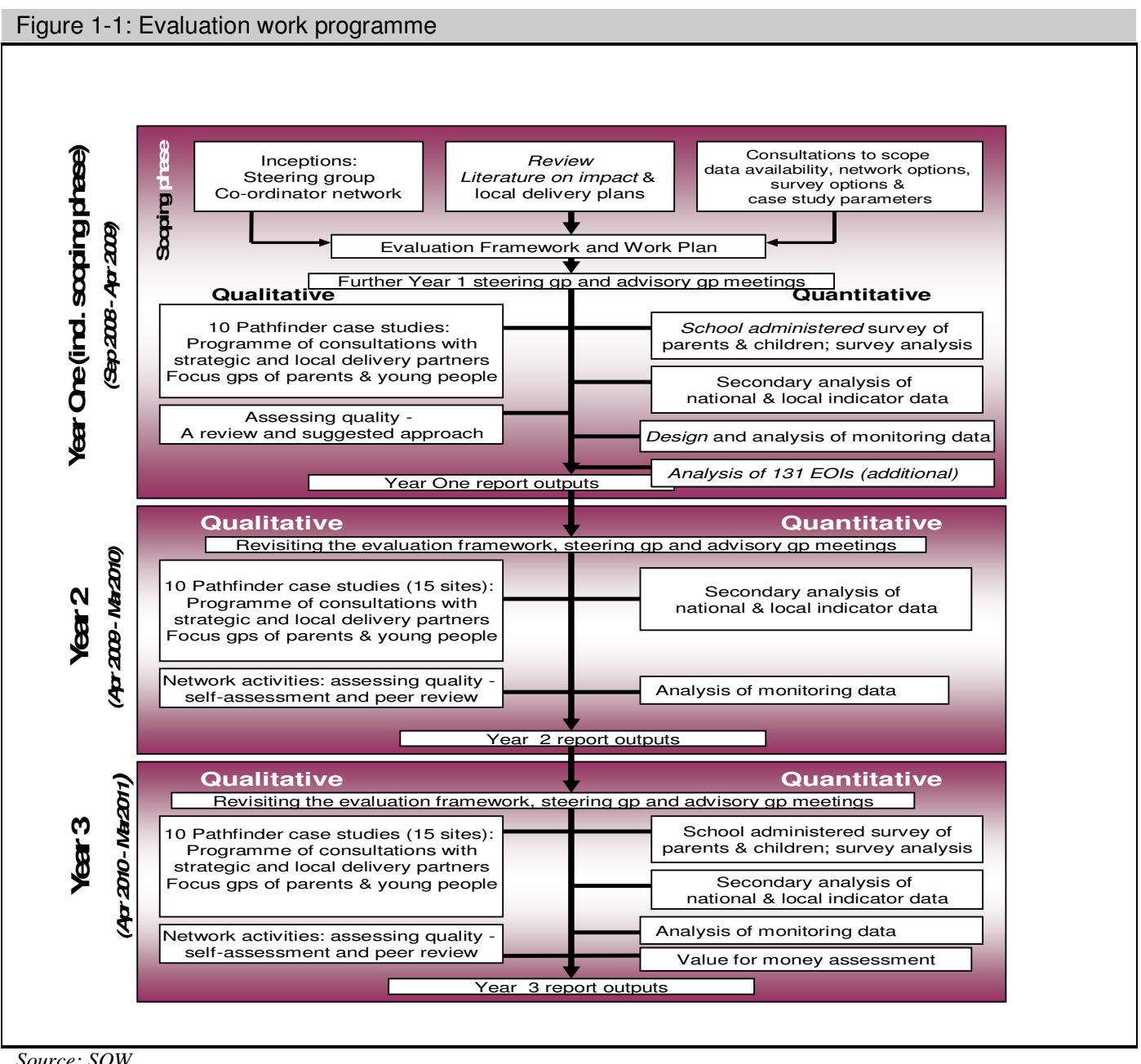

Source: $S Q W$

\section{Evaluation activities}

1.11 The first year of the evaluation (October - July 2009) was formative in orientation, investigating programme implementation in the ten pathfinder areas and identifying barriers to delivery and critical success factors. Most pathfinder activities in Year 1 focused on setting up management, governance and advisory functions and assessing need and auditing current provision. Evaluation activities during this period focused on constructing a pre-intervention baseline for the subsequent evaluation of change and impact, drawing on three main sources: secondary data analysis covering a wide range of contextual and outcome indicators; ten pathfinder case studies; and a baseline survey of more than 3,000 parents and 4,000 children in the pathfinder areas. (Following the termination of the evaluation contract, the planned follow-up survey will no longer take place.)

1.12 The second (and now final) year of the evaluation involved conducting a second wave of pathfinder case studies and analysing changes to contextual data, with a stronger focus on how activities planned in Year 1 were achieving results in Year 2. Over the two years, the case studies involved around 200 interviews and 28 focus groups with children, young people and parents. 


\section{Measuring success}

1.13 The measures by which the outcomes of FYT were to be assessed were derived from several sources, including the DCMS/DCSF evaluation terms of reference, the logic model designed for the evaluation, the DCMS/DCSF FYT Prospectus, and the ten pathfinder delivery plans for Year 1. They were developed with reference to the DCSF Every Child Matters outcomes and also research and evaluation evidence on the impact of cultural activities and interventions. The 16 primary output and outcome measures are summarised in Table 1-1.

\section{Table 1-1: Primary output and outcome measures}

- Increased participation in cultural offer

- Improved information and choice

- Increased mix and range of cultural activities in a local area

- New or improved partnership working

- Enhanced engagement with children and young people and families

- Enhanced awareness of what is on offer

- Enhanced awareness of one's own talent

- Enhanced knowledge and understanding of cultural forms

- Enhanced/shifts in perceptions and attitudes towards cultural activities

- Enhanced leadership characteristics in terms of identifying and supporting other young people

- Increased transferable skills/gaining new skills for producing cultural outputs

- Enhanced 'personal skills' as denoted by the QCA Personal Learning and Thinking Skills framework

- Enhanced enjoyment in cultural participation

- Enhanced coherence in cultural offer

- Enhanced or improved co-ordination in the cultural offer

- Improved quality of the cultural offer ( a combination of some of the outcomes above)

Source: $S Q W$

\section{This report}

1.14 This report provides an overview of findings from the first two years of the evaluation to the point of its termination in June 2010, during which time the ten FYT pathfinders began to deliver a universal cultural offer for children and young people in their areas. With regard to the five evaluation themes, the report presents baseline findings on participation in the ten areas, discusses early evidence for some of the outcomes listed above, considers the quality of the cultural offer made by the pathfinders, and considers the different pathfinder delivery models. Evidence discussed in the report has been gathered from a secondary analysis of national indicator data for the ten pathfinder areas, a baseline participation and attitudinal survey of parents and children conducted by Ipsos MORI, and two rounds of case study visits to the 10 pathfinders and their projects. In the absence of follow-up data, the report is silent on the overall impact of the programme, the relative effectiveness of different FYT delivery models, and value for money. 


\section{2: Local area characteristics and participation}

\section{Introduction}

2.1 An important element in FYT programme was the selection, from 141 expressions of interest, of ten pathfinders offering different area characteristics and delivery models, in terms of demand for and supply of cultural experiences, to test during piloting. This chapter considers evidence collected for the baseline (i.e. pre-programme evidence) area characteristics of the ten pathfinders and the levels and nature of participation in cultural activities, and identifies priorities for local action arising from the findings.

\section{Area characteristics}

2.2 Despite the intention to select pathfinders from areas with contrasting characteristics, national indicator data show that the ten pathfinders display similar demographic and economic area characteristics, as illustrated in the table below. They generally served large populations of children and young people, were relatively more deprived than the national average, and suffered from low levels of participation and attainment.

\section{Table 2-1: Demographic and socio-economic characteristics for the FYT pathfinders}

Pathfinders generally served large 0-19 populations; particularly large were FYT PUSH and Leicester and Leicestershire that spatially cover more than one local authority. Even where absolute populations were small (Telford and Wrekin and Bolton), they served large populations of children and young people as a proportion of their total populations.

Pathfinder populations were also generally more deprived than the national average, when measured by the Index of Multiple Deprivation (IMD) and Free School Meals (FSM) eligibility. This is particularly the case for Tower Hamlets in London.

Pathfinders experienced relatively lower levels of GCSE attainment. The gap in educational attainment between FSM and non-FSM pupils was particularly pronounced in Bolton.

Pathfinders also experienced relatively low levels of post- 16 participation in education and economic activity, and the proportions of 16-17 year olds not in education, employment or training (NEET) was a challenge for Bolton, Liverpool, Leeds and Telford and Wrekin in particular.

The 2008 and 2009 OfSTED Tellus survey of children and young people showed that, compared to $62 \%$ in England overall, a smaller proportion of children in pathfinder areas got involved in adult-led activities out of school in 2008. This was particularly the case for Tower Hamlets. There appeared to be unmet demand for music provision, cinema and theatre. However, participation in art, craft, dance, film and video making was higher than the national average.

Despite high levels of satisfaction with activities provided in their area, nearly half of the children responding to the Tellus survey in pathfinder areas thought that 'better activities for children and young people would improve their local area' and over half believed that they were not listened to in decisions about their local area.

Sources: $S Q W$ secondary analysis of national indicator datasets.

\section{Participation characteristics}

2.3 The Ipsos MORI baseline survey of parents and children highlighted some key characteristics of participation in, and attitudes towards, cultural activities among the 0-19 population in the ten pathfinder areas. 
2.4 Levels of participation ${ }^{6}$ were generally high among children in the pathfinder areas, with $89 \%$ of secondary-aged children participating in a cultural activity in the last seven days, either in school and/or out of school, in Spring 2009.

Figure 2-1: Types of cultural activities children participate in

\section{Types of cultural activities children participate in}

$\%$ Participating in any activity in last 7 days

$\%$ participating in each type of activity

Primary and secondary-age children
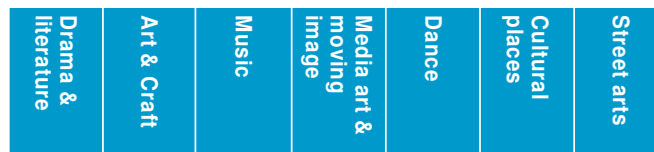

Out of school

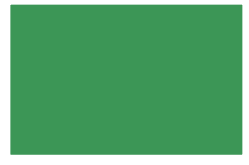

Secondary-age children

In school

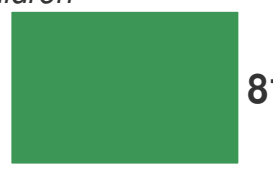

81

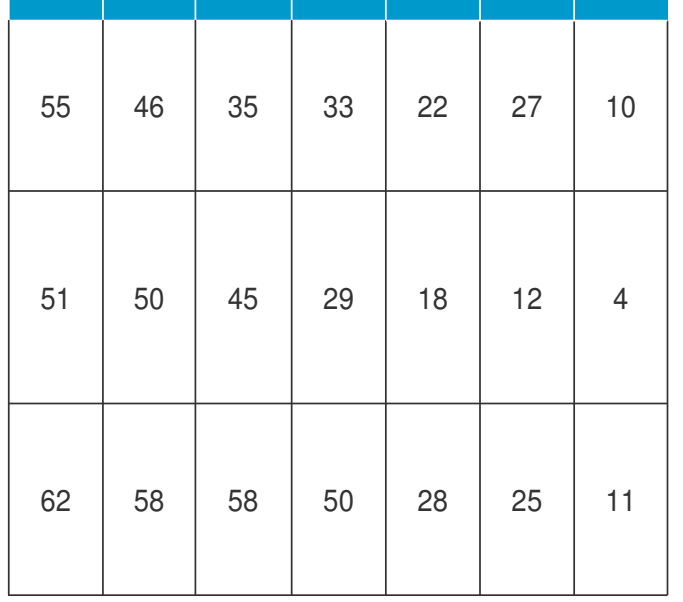

(in and out

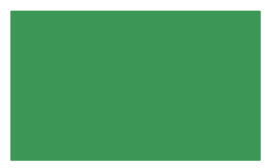

Base: All respondents. Secondary-age children (4,086); Primary-age children (3,316); March - June 2009.

Ipsos MORI

Source: Ipsos MORI baseline survey of parents and children, 2009

2.5 Almost two-thirds (62\%) of secondary-age children participated for five hours or more in any cultural activity. However, this finding masks the fact that fewer children of all ages participated in out-of school activities, and fewer secondary-age children participated in inschool activities:

- less than half (46\%) of primary- and secondary-age children spent five or more hours in out-of-school cultural activity in the week preceding their interview

- $\quad$ Three in ten $(29 \%)$ of secondary-age children spent five hours or more in the week preceding their interview in in-school cultural activity ${ }^{7}$.

\footnotetext{
${ }^{6}$ Due to the nature of self-completion surveys, at each question, there are a number of respondents who did not specify an answer. This affected data from the pupil survey in particular. As a result, an imputation exercise was undertaken by Ipsos MORI to address missing responses on the pupil-level data. Children are classified as a 'participant' in an activity if they/their parent ticked at least one of the listed activities within that cultural form, and specified that they spent at least 30 minutes participating in that form of activity in the last seven days. The percentages presented for the five hour measure include all respondents for whom comprehensive timings are available.

${ }^{7}$ Note that parents of 5-10 year olds were not asked about their in-school cultural participation.
} 


\section{Amount of time spent doing cultural activities in past week}

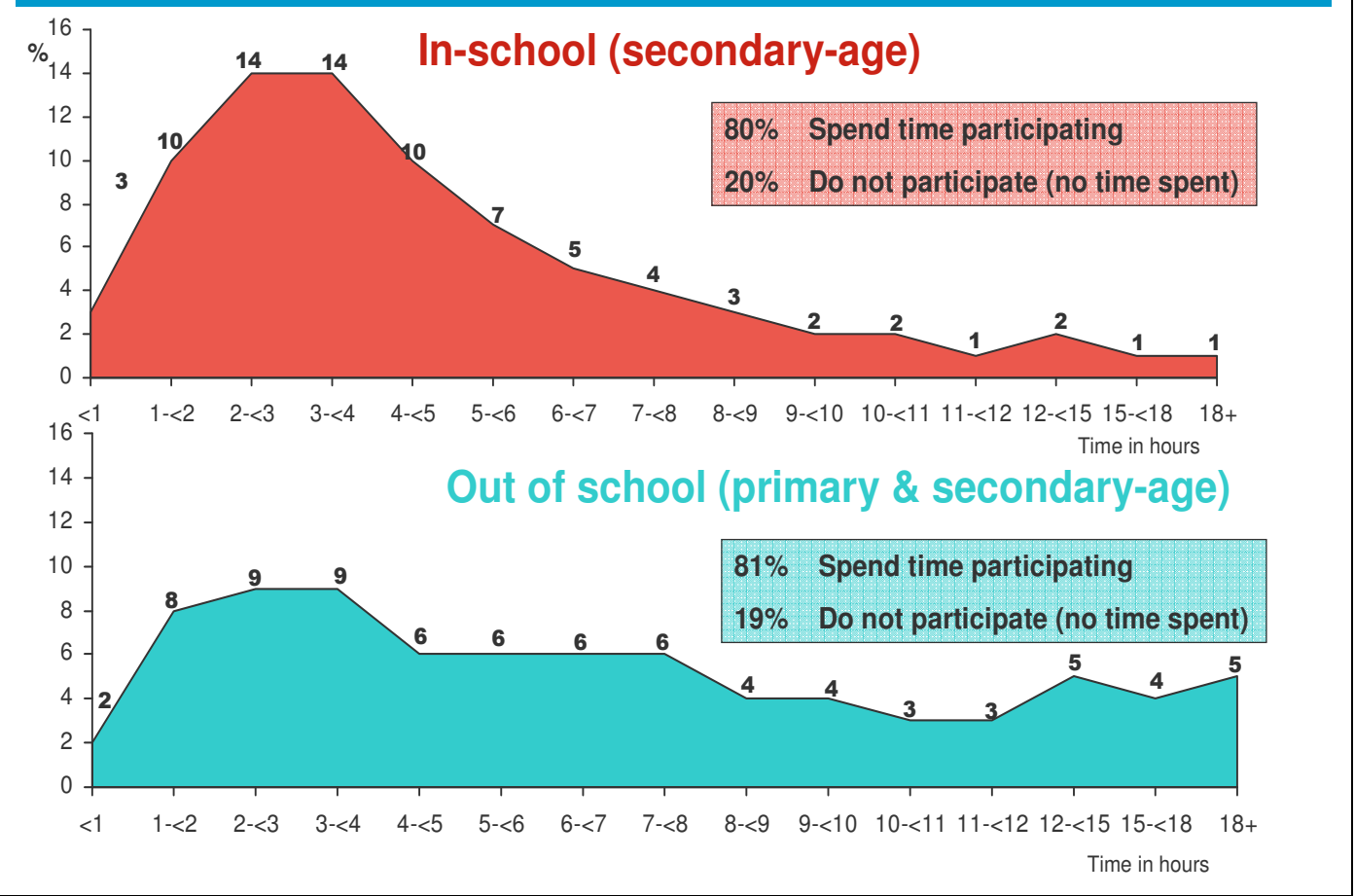

Source: Ipsos MORI baseline survey of parents and children, 2009

Figure 2-3: Amount of time spent doing cultural activities in past week

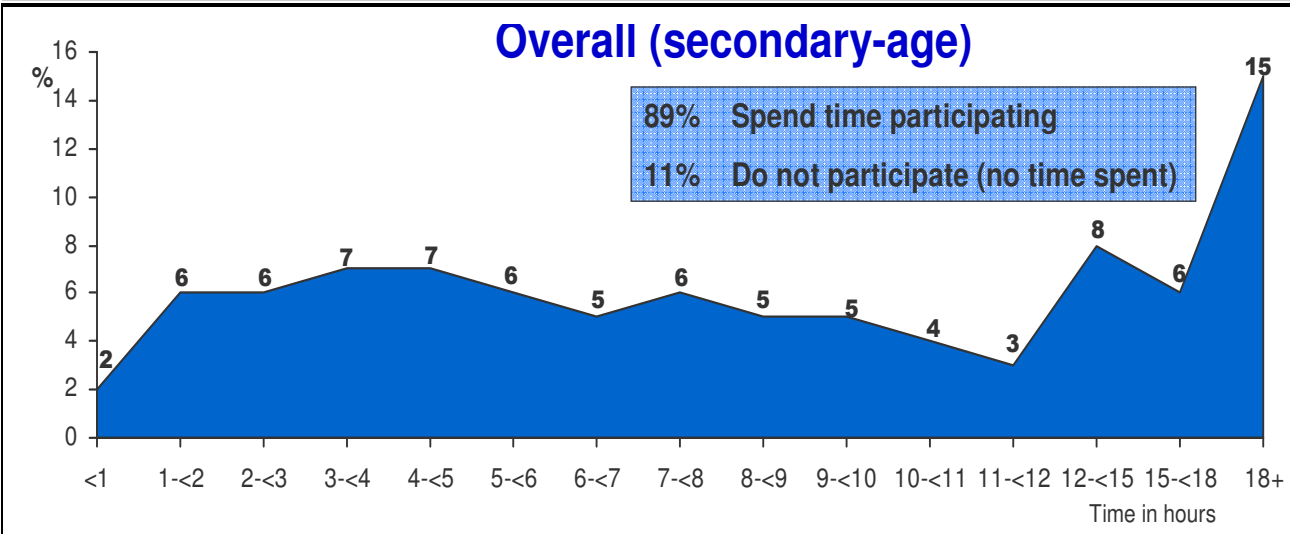

Base: All respondents for whom comprehensive timings are available. In-school $(4,029)$; Out of school $(6,534)$; Overall $(3,945)$ March - June 2009.

Ipsos MORI

Source: Ipsos MORI Wave 1 survey of parents and children, 2009

2.6 Primary-age children tended to spend more time doing cultural activities outside of school than secondary-age children; over half (54\%) of primary-age children spent five hours or more participating in cultural activities outside school compared with two-fifths (40\%) of secondary-age children. The amount of time spent participating seems to decline with age: it is highest among children in school years 1-3 and lowest among children in school years 10-11. 


\section{Amount of time spent participating outside of school by school and year group}

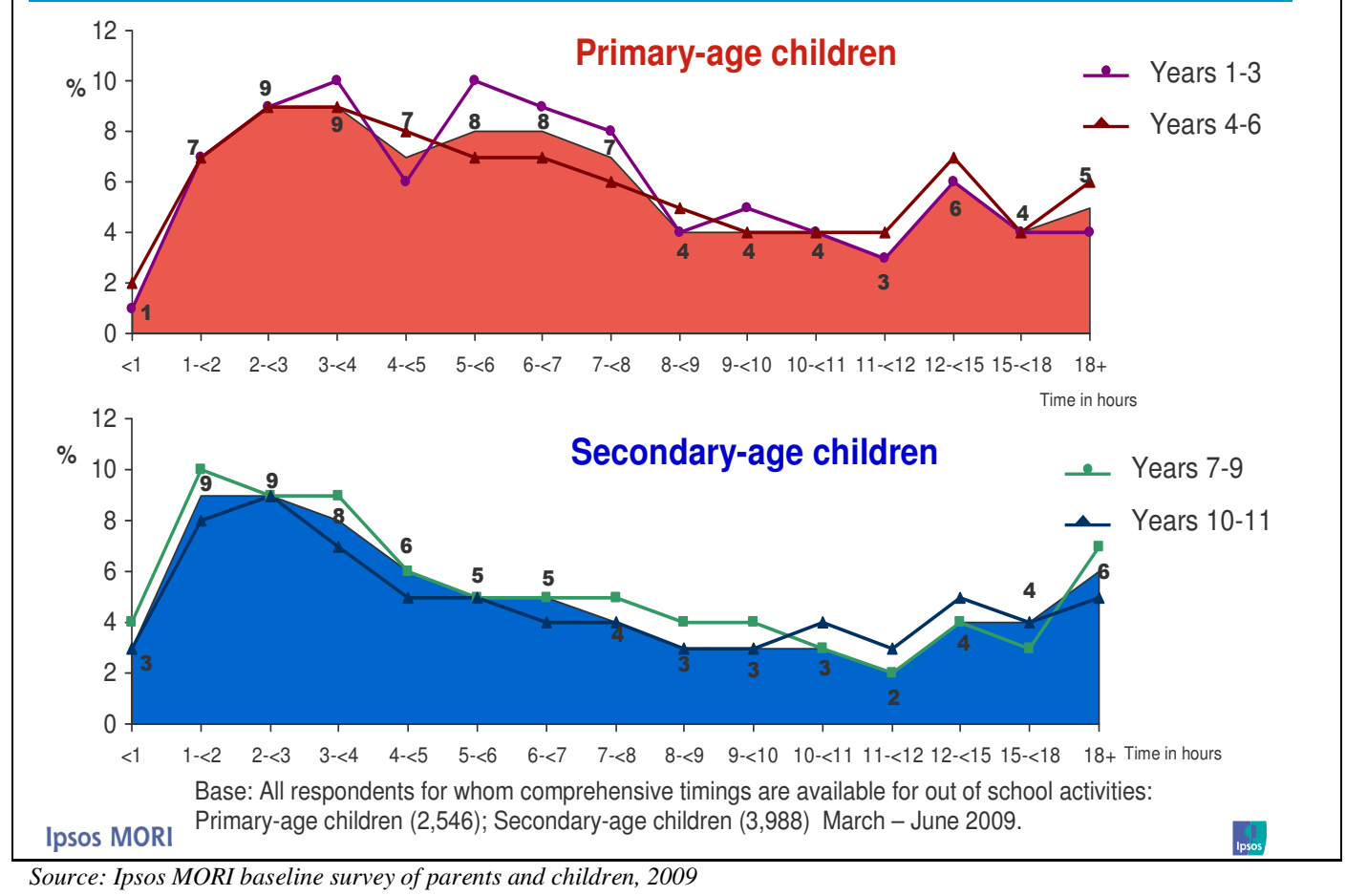

2.7 Levels of participation varied among specific groups. Older boys, ethnic minority groups and those eligible for free school meals were least likely to participate in the last seven days when interviewed, while younger children, girls and those from White and less deprived backgrounds were most likely to do so.

2.8 Interestingly, attitudes to participation were associated with current levels of engagement. Where children were already engaged, the experience seems to have encouraged them to aspire for more and better provision:

- $\quad$ High participators (as defined by those spending five hours or more per week) were most likely to want to participate in the future, and also to voice opinions about the quality of the current offer, than low participators (those spending 30 minutes or less)

- $\quad$ Low participators were more content with the current offer (for example, parents of primary-age children from Asian backgrounds), yet parents of primary-age children with a SEN/disability were significantly more likely than other parents to want a 'lot more' and/or better activities, despite being relatively low participators.

2.9 A majority of parents and children wanted to participate more in the future, but thought that the quality of the current offer and information about what was available in their area could be improved. 
2.10 Lack of information was most likely to be cited as the reason for not spending more time participating in cultural activity. Neither parents nor children rated the quality of information available highly. Friends, family and word of mouth were reported as the key sources of information, particularly amongst secondary-age children, but parents of primary-age children would prefer more formal written information, such as school newsletters, as would parents with no qualifications and those whose children are eligible for FSM. Access to cultural activity was also a particular issue for lone parents of primary-age children and also children who are eligible for FSM.

Figure 2-5: Quality of, and demand for, local cultural activities

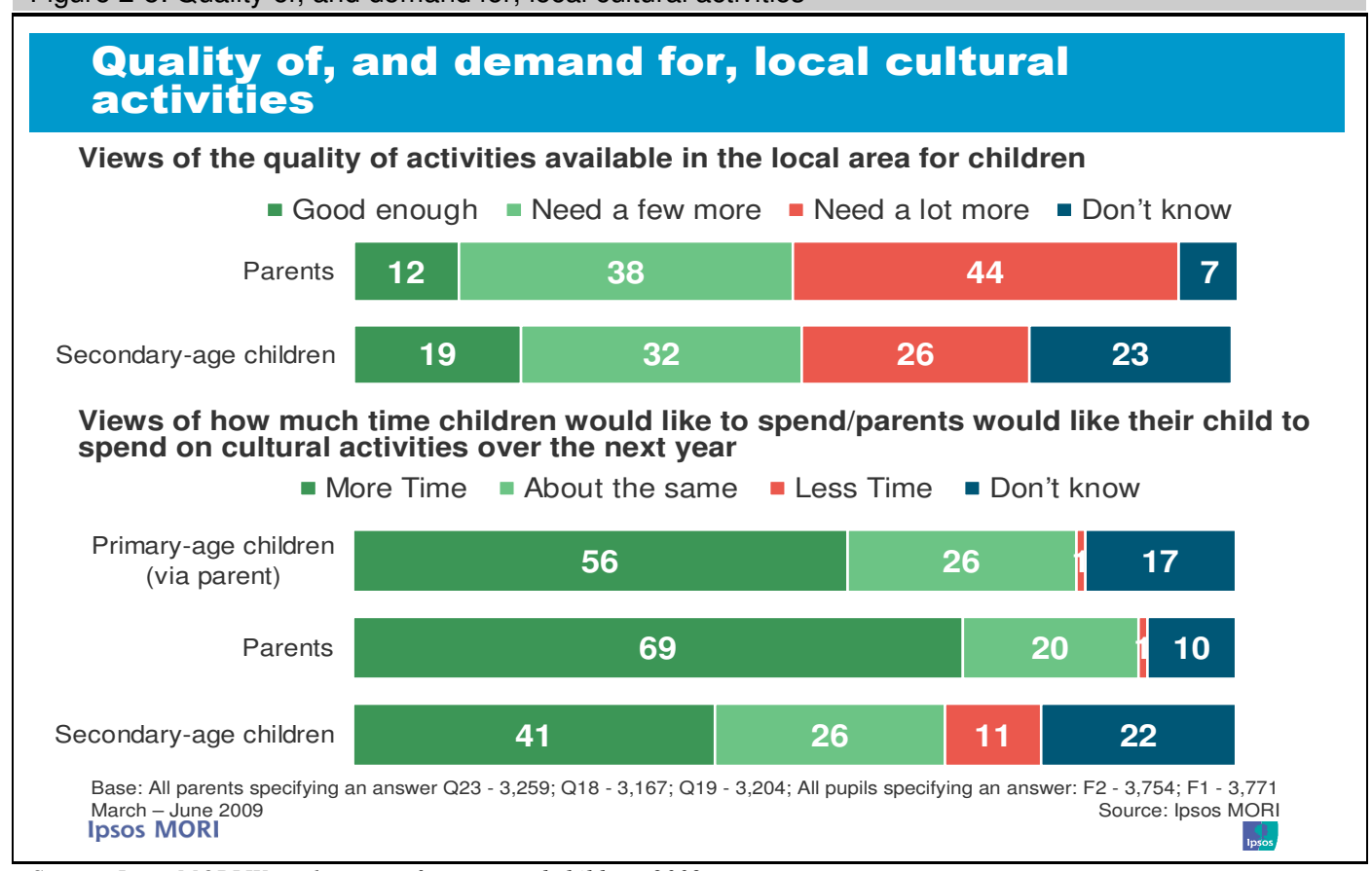

Source: Ipsos MORI Wave 1 survey of parents and children, 2009

2.11 Parents and children did not feel they are listened to. Only 19\% of parents felt that they were listened to a great deal or fair amount in the planning of local cultural activity, and only $14 \%$ felt their primary-age child was listened to. Secondary-age children were slightly more likely to feel listened to, but fewer felt listened to than have actually taken part in some kind of consultation activity (29\% said they were listened to at least a fair amount and $43 \%$ gave their views in some way in the last 12 months).

2.12 Parents and secondary-age children were positive about their creative talents, but girls were significantly more likely to consider they have talent than boys; however, boys thought they had stronger interpersonal skills. Nevertheless, less than half (49\%) of secondary-age children said they knew a fair amount about the range of careers open to them in the creative and cultural industries.

2.13 Results varied among the different pathfinder areas. Shepway and PUSH had the highest baseline levels of participation overall for secondary-age children. In contrast, baseline participation for secondary-age children was lower than average in North Somerset and Leicester and Leicestershire. For primary-age children, Leicester and Leicestershire had the highest, and Tower Hamlets had lowest levels of participation. Secondary-age children in 
Tower Hamlets and parents of primary-age children in Liverpool were most likely to say the existing cultural offer needed to be improved. Parents in Liverpool were most likely to say that information about cultural activities in their area needed to be improved, along with secondary-age children in Tower Hamlets.

\section{Conclusion}

2.14 These findings have the following priorities for area-based planning for demand-led cultural provision for children and young people:

- Increase participation amongst specific groups, specifically older children, boys, those from ethnic minority backgrounds and SEN/disability, and those from lowincome and single-parent households; this crucially means that besides increasing provision overall, proactive engagement work is required to narrow the gap between experiences, participation and expectations among the high and low engagers

- $\quad$ Shift attitudes about participating in culture among groups that tend to be less engaged and less vocal about their needs and interests

- $\quad$ Encourage peer recommendation and word of mouth for secondary-age children, who tend to be reluctant to go unless they know other children who are attending, and harness the potentially powerful role that schools can play in this regard

- $\quad$ Strengthen how the offer is communicated to children and young people and their parents, using schools to channel this information

- $\quad$ Engage parents and children in planning and designing the local offer

- $\quad$ Promote progression to careers in the cultural and creative industries, in order to inform decisions about progression to further learning and employment

- Improve the quality of the cultural offer. 


\section{3: Area-based delivery models}

\section{Introduction}

3.1 This section describes models deployed by pathfinders for delivering FYT, based on reviews of pathfinder delivery plans and case study consultations with pathfinder teams, and other local partners and stakeholders. Delivery models typically feature one lead organisation that sets up specific management and governance structures and identifies key strategic and delivery partners. Models are also defined by what the focus for delivery is - targeted or universal provision, and new or extended existing activities. These are the main variables in the different types of models developed by the pathfinders, and they operate at a high level below which fragmentary and localised differences (e.g. which new activities, how are governance functions discharged?) reduce the possibilities for meaningful comparative analysis. Moreover, in the absence of follow-up post-programme evidence (curtailed by the termination of the evaluation contract), it is not possible to assess the relative effectiveness of different delivery models in improving the cultural offer for children and young people in the ten pathfinder areas.

\section{Pathfinder delivery models}

\section{Management, governance and partnerships}

3.2 A majority (seven) of the ten pathfinders were led by local authorities as the accountable body, with the rest led by a cultural sector organisation working closely with the relevant local authority within the management structure. The core partnership for a majority of the ten pathfinders included three key members: local authorities; cultural organisation; and schools.

3.3 There were variations in the extent and ways in which cultural organisations were engaged in designing and delivering the programme where a local authority was the lead body. The influence that the core partnership exerted over the local delivery of FYT varied depending on whether these partners belonged to the core management team, were represented on a project steering group or advisory board, or belonged to the wider group of local stakeholders, networks and delivery partners.

3.4 The management structure in most pathfinders was characterised by an operational or delivery arm and a separate accountable body. The former typically comprised a project director and manager, with support from a range of key staff, including delivery officers (e.g. area co-ordinators or development officers) who work on the ground to deliver activities. In some cases, these were established posts in the lead or partner organisations. Potentially such arrangements could become embedded within existing organisational structures, enabling resource efficiencies and encouraging sustainability.

3.5 A pathfinder delivery team (typically a director or manager and delivery officers) would usually report to the lead accountable body, which was supported by a steering group or a project board. The membership of such groups included representation from the accountable 
body as well as key strategic partners from cultural organisations, schools, and local authorities. The role of the accountable body was to provide strategic and artistic direction, develop and maintain good relationships with partners and stakeholders, and also oversee progress in delivering the programme and ensuring effectiveness of the delivery team.

3.6 In one case where the pathfinder (Liverpool), was being led by a cultural consortia (Liverpool Arts and Regeneration Consortia (LARC)) the management team was hosted by one of the consortia members (National Museums Liverpool) that was also the accountable body. The pathfinder had two other layers of governance: a Management Group that identified and prioritised the strategic focus for the FTY programme for Liverpool; and a Programme Group that was responsible for the overall artistic direction and commissioning of activities.

3.7 Other governance structures took the form of a Cultural Stakeholders Group (Tower Hamlets) and a Local Development Group (Telford), both of which provided cross-cultural and other expertise and played the role of shaping the local FYT offer.

3.8 Sitting alongside these structures were groups of representatives from key partners providing strategic advice rather than executive functions. These groups also initiated debate, challenged the functions and direction of the management and governance teams, and engaged a wide range of partners and stakeholders involved in delivery of cultural activities. In some cases (e.g. Leicester and Leicestershire, and Shepway), such groups also provided support and advice on which activities should take place and how their success should be judged.

3.9 The management and governance structures also had in their delivery models formal and informal mechanisms for engaging children and young people in aiding the design and delivery of provision. In some cases (Customs House, Leeds, Tower Hamlets), these groups were intended to feed directly into the decision-making processes around design and delivery. In others (Leicester and Leicestershire, Liverpool, PUSH, Shepway) they played a more advisory and consultative role, but still were positioned alongside the management arrangements to allow for direct influence and feedback.

3.10 It should be noted that two pathfinders (Shepway and Tower Hamlets), experienced significant changes to their management structures during the programme. Shepway experienced considerable turmoil in the management team during the first year, with the project director on leave for extended periods of time and finally leaving the programme in late November 2009. Although the director was not replaced, the project coordinator for the programme was given an enhanced level of responsibility, and the Chair of the project board and Artistic Director of the Creative Foundation dedicated more time for the remainder of the programme. Similarly, Tower Hamlets experienced significant staff changes during the second year. These included the departure in December 2009 of the Service Head for Young People and Learning, who acted as the Chair of the project board, and the pathfinder manager, who coordinated the pathfinder delivery team and activities. The pathfinder manager was replaced in February 2010 and at the point of conducting the evaluation, the Chair of the project board had not been replaced. However, the delivery team stated that the new Service Head for Young People and Achievement was to take up this post at the end of April 2010. These changes had a significant impact on programme delivery in these areas, and underline the importance and securing the continuing contribution of key delivery staff. 


\section{Sustained structures and systems}

3.11 Pathfinder approaches to ensuring the sustainability of FYT beyond the programme funding period were closely linked to the lead organisation, which in most cases afforded opportunities for embedding delivery structures and mechanisms in ways that would sustain the positive impact of the programme. For example, for many local authority-led pathfinders, programme structures were designed to embed the cultural offer within existing structures for managing and delivering services for children.

3.12 Many pathfinders had ensured at the end of Year 1 that this was the case, in order to avoid duplication of effort, enable joined-up working and ensure alignment of strategic priorities. Others had built on the inherited structures of existing initiatives to achieve economies of scale and scope. The following examples illustrate these approaches:

- The programme management structure in Tower Hamlets comprised three groups, the Project Board, the Commissioning Group and the Cultural Stakeholder group, each of which fed directly into existing structures within the council and the Local Strategic Partnership. The new structures sat within the council's Children's Services team to ensure the programme was aligned to strategic objectives by forming linkages with the Early Years, Young People and Learning teams and also the Community Culture and Leisure teams. Stakeholders hoped that over time the FYT structures would be absorbed within these parts of the council.

- The cultural organisation-led pathfinder, Liverpool, had a management structure with representation from all key partners. The ultimate responsibility for the pathfinder sat with LARC Chief Executive Officers. ${ }^{8}$ The FYT management group comprised Heads of Service from the local authority alongside LARC lead officers and representatives from Creative Partnerships Merseyside, the Liverpool Culture Company and National/Regional Development Groups.

- In North Somerset, a conscious decision was taken to limit the creation of new posts for FYT whilst supporting programme delivery through key staff employed in the Children and Young People's Services, and to fill a perceived gap in skills around commissioning of cultural activity by employing a 'Cultural Programmer'. The work of the pathfinder was intended to dove-tail FYT activities into other initiatives and activities that were already being undertaken, thus allowing the pathfinder to focus resources elsewhere on initiating new projects.

3.13 Some pathfinders took innovative measures when designing and delivering the offer to ensure that partnerships could be sustained after the FYT programme ended. North Somerset's 'legacy projects' have potential to become cornerstones in a future cultural offer because they are not dependent on FYT funding. The Tower Hamlets consortia-based approach and focus on capacity building could enable the cultural offer to be mainstreamed and developed within existing service provision. In Leicester Shire schools, the cultural offer was made within a wider range of activities delivered through Extended Schools and for Gifted and Talented

\footnotetext{
${ }^{8}$ LARC comprises the National Museums Liverpool, the Royal Liverpool Philharmonic, the Bluecoat, FACT, Liverpool Biennial, Liverpool Everyman and Playhouse, Tate Liverpool and the Unity Theatre.
} 
pupils, with additional funding sought from the Building Schools for Future programme and Community Service Pilots.

\section{Delivering cultural provision}

3.14 The FYT sponsor departments, DCMS/DCSF, stipulated that pathfinders were required to demonstrate two things when commissioning provision in their areas:

- that they were delivering a mix of new provision and extending existing provision, both in terms of funding but also in useful, innovative ways by improving coordination and coherence

- $\quad$ that they specified their target groups, in terms of providing for all 0-19 year olds in their areas or being more selective and targeting specific sub-groups such as young people who are NEET.

3.15 At the end of Year 2, it was clear that a majority of the pathfinders had introduced a "progressive universalism" approach to delivering the cultural offer (see Table 3-1 below). Progressive universalism is defined in this context as a cultural offer for all children and young people (i.e. universality), with more support to access provision for 'low participators' to minimise barriers to their participation (i.e. spatial or demographic targeting).

3.16 Pathfinders had been targeting disadvantage in specific geographical areas (particularly areas of multiple deprivation or rural isolation), often combining this with specific socio-economic characteristics that impede access to cultural activity. Where pathfinders had adopted spatial targeting only (e.g. Bolton and Shepway), they were still targeting all 0-19 year olds within their target areas. Here the intention was to adopt universality once the targeted hard-to-reach groups and particular areas have been addressed by the programme.

3.17 Furthermore, as initial projects were tested in Year 1, pathfinders sharpened their focus further in Year 2, some towards a more universal offer (e.g. Telford) and others taking a more targeted approach (e.g. Customs House).

Table 3-1: Universality and targeting of cultural provision

\begin{tabular}{|c|c|c|c|}
\hline & Universal & Geographical targeting & Demographic targeting \\
\hline Bolton & & $\checkmark$ & \\
\hline Customs House & & $\begin{array}{l}\quad \checkmark \\
\text { Focusing on areas with } \\
\text { high rates of teenage } \\
\text { pregnancy, anti-social } \\
\text { behaviour and poor mental } \\
\text { health }\end{array}$ & $\begin{array}{ll}\checkmark & \text { Young } \\
\text { people } \\
\text { NEET, } \\
\text { looked after } \\
\text { children } \\
\text { (LAC) }\end{array}$ \\
\hline Leeds & $\checkmark$ & $\checkmark$ & LAC \\
\hline $\begin{array}{l}\text { Leicester and } \\
\text { Leicestershire }\end{array}$ & $\checkmark$ & $\checkmark$ & $\begin{array}{l}\text { Black and Minority Ethnic } \\
\text { communities, children and } \\
\text { young people with disabilities, } \\
\text { areas of multiple deprivation }\end{array}$ \\
\hline Liverpool & & $\begin{array}{l}\text { Areas of multiple } \\
\text { deprivation }\end{array}$ & Young people NEET \\
\hline
\end{tabular}




\begin{tabular}{|c|c|c|c|}
\hline & Universal & Geographical targeting & Demographic targeting \\
\hline North Somerset & $\checkmark$ & & $\begin{array}{l}\text { Moving away from school } \\
\text { provision to reach those who are } \\
\text { disengaged }\end{array}$ \\
\hline PUSH & $\checkmark$ & $\checkmark$ & $\begin{array}{l}\text { Young people NEET, children } \\
\text { and young people with } \\
\text { disabilities, young offenders, } \\
\text { BAME, young carers, looked } \\
\text { after children }\end{array}$ \\
\hline Shepway & & $\checkmark$ & $\begin{array}{l}\text { Looked after children, children } \\
\text { and young people with } \\
\text { disabilities and additional needs, } \\
\text { young people NEET, BAME } \\
\text { communities }\end{array}$ \\
\hline Telford and Wrekin & & $\begin{array}{l}\text { Extending reach beyond } \\
\text { geographical clusters }\end{array}$ & $\begin{array}{l}\text { Children with disabilities, early } \\
\text { years and families }\end{array}$ \\
\hline Tower Hamlets & $\checkmark$ & $\checkmark$ & $\begin{array}{l}\text { Focus on families (including } \\
\text { parents) who are under- } \\
\text { represented in accessing } \\
\text { cultural activities }\end{array}$ \\
\hline
\end{tabular}

Source: $S Q W$ analysis of pathfinder delivery plans and case studies.

3.18 A combination of adding new provision, extending existing provision and/or improving the co-ordination and coherence of existing provision was adopted by most pathfinders but in different ways. Those starting with less cultural activity tended to focus on predominantly new provision (Bolton, Customs House, North Somerset), whilst those starting with more current cultural activity emphasised extending or better co-ordinating provision as well (Liverpool, Leicester \& Leicestershire, Tower Hamlets). The other pathfidners (Leeds, PUSH, Shepway and Telford and Wrekin) offered a combination of new and existing provision, with an added focus on co-ordinating provision as well. In these ways, pathfinder delivery models responded to current levels of supply and participation in cultural activities in their areas.

\section{Conclusion}

3.19 The vision for a demand-led, area-based and high-quality cultural offer conceived by the ten FYT pathfinders reflects the baseline survey findings discussed in the previous section improving access, raising aspiration, strengthening co-ordination of existing provision, filling gaps with new activities, offering an appropriate mix of provision and focusing on new activities. This shows that the pathfinders had identified and planned provision for meeting the needs of children and young people in their areas. Although the organisation leading the pathfinder had an effect on how and to what extent cultural and educational partners were engaged in the design and delivery of FYT locally, it is clear from the pathfinder delivery models that this was less of an issue when it came to the vision of a cultural offer. Most pathfinders had, or were intending to adopt, a progressive universalism approach that places greater emphasis on targeting the most vulnerable groups, and delivering an appropriate mix of provision, rather than simply 'adding' new cultural activities without reference to current cultural provision or the needs of particular groups of children and young people in their areas. 


\section{4: Pathfinder effectiveness}

\section{Introduction}

4.1 This chapter assesses the effectiveness of FYT programme delivery in the ten pathfinder areas, drawing from case study evidence contributed by local stakeholders and delivery partners. It considers the effectiveness of programme and partnership arrangements, and targeting different types of provision.

4.2 Pathfinder effectiveness can be judged by assessing the presence and strength of key indicators that drive successful local FYT programme implementation, including the following:

- $\quad$ sustained management, governance and partnership structures in line with pathfinder aims and objectives

- $\quad$ shared vision and buy in among partners and stakeholders

- delivery mechanisms that ensure the sustainability of the programme

- careful research and auditing of need among target populations, and the responsiveness of programme delivery mechanisms to meeting identified needs

- $\quad$ established procedures for engaging children and young people which directly inform programme design and delivery

- the scale, range and reach of the cultural activities delivered

- $\quad$ improved coherence and co-ordination of the cultural offer across the local area.

4.3 Not all of these are expected to be evident in the early stages of programme development and delivery covered by the evaluation before its termination. However, the initial years of any programme intervention can provide important insights into 'what works' in programme delivery and what needs to be improved, highlighting good practice as well as identifying lessons for generating positive outcomes for children and young people.

\section{Effective practices}

\section{Challenges}

4.4 Case study evidence shows that the ten pathfinders faced a number of challenges to the effective delivery of a universal cultural offer for children and young people in their areas, including the following:

- Organisational change - for most pathfinders, programme management and governance arrangements took longer to establish than they had anticipated; whilst a majority managed to establish and maintain these into the second year, this was not the case for the two pathfinders that experienced significant organisational changes 
which affected the effectiveness of their delivery and (in one case) partner disengagement

- $\quad$ Focusing on demand - as a high-profile national programme, FYT attracted widespread interest from a host of potential delivery partners with a wealth of possible supply-side solutions, which did not necessarily fit with pathfinders' planned provision on the basis of local needs and priorities

- $\quad$ Engaging partners - by Year 2, most pathfinders had succeeded in securing the partners they needed for effective delivery, however a small number of pathfinders experienced significant difficulties engaging partners; this was particularly the case with schools, which either did not immediately see the relevance of FYT for curriculum delivery, or otherwise had more pressing 'school improvement' priorities

- $\quad$ Engaging children and young people - the practicalities of recruiting children and young people and ensuring their views were considered throughout the programme cycle proved to be a challenge for several pathfinders; whilst the aspiration of Year 1 was to have formal and informal mechanisms in place to allow young people to design and help deliver the offer, by Year 2 not all pathfinders had been able to implement their engagement activities as planned.

4.5 In the following sections, we discuss how pathfinders tackled these challenges.

\section{Leadership, management and governance}

4.6 There was no clear evidence from the evaluation on whether pathfinders led by local authorities or cultural organisations were more effective. Admittedly, post-programme evidence of change on the key output and outcome measures identified in Table 1.1 is required to assess the effectiveness of these two types of pathfinder leadership. However, on the basis of the mostly baseline and process evidence collected up until the point at which the evaluation was terminated, it can be said that both types of pathfinder leadership have their advantages and were effective in different ways. Most likely, local circumstances will decide which of the two approaches is most effective in addressing local needs.

4.7 In the former, the FYT management and governance arrangements could be firmly embedded within existing local authority children and young people's services. The management team had better access to, and therefore stronger links with schools. In the latter model, the pathfinder management team had direct links with, national and local cultural providers, and was less exposed to political change and pressures.

4.8 Case study evidence suggests that local authority pathfinder leadership can facilitate embedding management arrangements for the cultural offer within existing local authority structures whilst sustaining strong cultural sector engagement. For example, in North Somerset, a cultural programmer led FYT activities and provided exceptional leadership and vision for the area, and in Bolton the cultural change team was the main route for engagement with teachers and cultural organisations and area-based teams collaborated to deliver largescale activities. Even amongst the few (three) pathfinders led by cultural organisations, good examples exist of embedding FYT management arrangements within existing local authority 
structures whilst facilitating strong engagement with the cultural sector. For example, the Liverpool pathfinder was strongly connected to local authority teams in three areas in order to target young people effectively:

- In St Helens, the Area coordinator was based in the Children's Services Directorate, along with Healthy Schools team historically quite removed from the council's Art Service

- In Liverpool, the coordinator sat within the Arts, Heritage and Participation team, in Culture Liverpool, the arts business unit of the City Council

- In Knowsley, the coordinator sat within the South and North Kirby area team, a neighbourhood based team.

4.9 Strong leadership and management was a key requisite for successful delivery of the wide range of functions pathfinders engaged in as enablers, coordinators, brokers and innovators for the cultural offer. A skilled, experienced team with good leadership attributes and structures that enable these attributes to be further enhanced via CPD worked best in engaging partners and achieving buy in from key stakeholders. These views were strongly expressed during case study consultations, supported by an observation made by the evaluation team that a consistent and stable project leadership enables a smooth and timely delivery of activities. In a small minority of cases (Shepway and Tower Hamlets) where pathfinders experienced significant changes in their management and leadership, project delivery was disrupted and delayed as a result.

4.10 Local stakeholders consulted during the case studies were very positive about the effectiveness of pathfinder leadership and management. In Leicester and Leicestershire 'the team was slick and effective, challenged the norm', while in Telford they provided 'highly efficient support, setting plans and milestones for partners'. The Tower Hamlets delivery team was regarded as innovative in their approach despite the challenges it faced engaging stakeholders inside and outside the council. The area co-ordinators were seen as a driving force in Liverpool in delivering a coordinated and coherent offer across the three priority neighbourhoods. The core team in Telford was also commended for showing strong leadership qualities and acting as a catalyst to raise the level and quality of provision and instigate new partnerships. Stability within a management team was also a contributing factor to its success.

\section{Partnerships and networks}

4.11 Pathfinders were effective in building capacity and working in partnership to deliver the FYT programme. New and improved partnerships had been established. Many stakeholders and partners acknowledged this effort positively and noted it had generated joined-up working amongst providers and stakeholders and improved co-ordination of the cultural offer that would not have taken place without the programme.

4.12 The setting up of formal structures helped set appropriate and useful information and strategy sharing protocols among partners and stakeholders. Once these protocols were established, it 
enabled partners to coordinate their activities and be better informed about where and how to target their offer.

4.13 For example, a major development for the Leeds pathfinder had been the launch of the Breeze Culture Network, a professional networking platform for organisations and individuals seeking to develop cultural opportunities for young people in the city. The online network shares contact details of schools in the area with practitioners and arts organisations who are looking to connect directly with schools and young people. Members can also access a citywide event planner and date diary, and can publish events and activities on the Breeze Leeds website, which has over 120,000 young people signed up as members. Consulted partners and stakeholders noted that the network was well suited for promoting a clear picture of the city's cultural offer. The central registration and safeguarding processes, the directory of contacts and the networking features had contributed to improving the coordination of the city's cultural offer, and cultural organisations and practitioners were keen to be involved and make the new tools work effectively.

4.14 Formal structures also took the form of advisory groups that sat alongside the management and governance structures, and helped guide the delivery of the offer on a day-to-day basis. For example, the Customs House North and South Tyneside Working Groups included teachers, cultural organisations and Connexions, who provided a sounding board for FYT activity. They also made connections between partners and provided them with a supportive network.

4.15 Links with national partners were also considered to be vital in establishing effective and sustained partnerships through reputational effects. This underpinned successful efforts by Customs House to work with national cultural organisation as delivery partners in Year 2, including the Royal Opera House and Sadler's Wells. The Telford pathfinder also secured the involvement of the Royal Shakespeare Company Learning and Performance Network, enabling access to a wide range of regional partners, and Liverpool's LARC partnership involving national partners was viewed by stakeholders as providing appropriate representation and robustness to the programme in the area.

4.16 Continuing professional development (CPD) for practitioners and providers was seen by stakeholders and partners as a key indicator for effective partnership working. Leeds had Active Learning Modules for teachers and artists, and Tower Hamlets had included a CPD element in all strands of their programme work. Indeed, a significant amount of pathfinder activity in Year 2 sought to facilitate CPD activities in local settings and schools, to secure capacity to deliver the activities beyond the programme funding period. In another example, an active learning CPD project in Leeds aimed at primary teachers and creative practitioners offered opportunities for them to work together and design ideas for inclusion in the classroom.

4.17 One pathfinder was in a unique position of marrying three different local authorities covering disparate target groups and differing set of policy priorities. In a number of ways, PUSH ensured that the pathfinder's management and governance arrangements allowed for this FYT partnership to function efficiently. These included a series of strategic discussions to ensure that learning and outcomes from the programme are embedded in future planning, and 24 
cross-PUSH programmes with the capacity to inform and influence delivery of cultural activities across the three local authorities in the PUSH area.

\section{Partner schools}

4.18 The FYT programme created opportunities for schools to participate as delivery partners. A distinction should be drawn between the whole-school focus of Creative Partnerships and the stronger focus on individual children and young people in FYT. Schools in FYT were mostly involved either as delivery partners or represented on operational advisory groups. They did not lead pathfinders, and tended not to contribute to strategic decisions about the overall offer in general. ${ }^{9}$ Yet schools still had an important role to play in communicating the cultural offer to parents and children and delivering in-school activities.

4.19 Despite these contributions, several pathfinders had difficulty in engaging school partners. In one case (Bolton), local specialist schools reportedly saw little benefit in participating. Elsewhere (Leeds) case study consultees reported that some schools, particularly those in the most deprived areas, were too occupied with existing school improvement initiatives to engage with FYT. In addition, teachers were often too busy with regular curriculum work to take up the cultural activities offered through FYT, particularly when access to these opportunities involved some sort of application or bidding process. In the case of Tower Hamlets, head teachers supported the aims of FYT but they struggled to see how it aligned with the curriculum and thus felt unable to prioritise it over other activities they offered to their pupils.

4.20 Faced with barriers such as these, pathfinders tended to work with the more willing schools, or focussed their work on out-of-school activities. For example, the PUSH pathfinder was clear at the outset that FYT was an out-of-school programme. However, this did not stop the pathfinder from working with local schools' extended services, as did Telford and North Somerset. Case study evidence shows that partners felt that such activities were having a positive impact, and helped to sustain cultural activities within wider extended schools activities.

4.21 Several pathfinders demonstrated what could be achieved through close partnership working with schools in delivering the offer, as the following examples illustrate:

- $\quad$ Using Cultural Organisations to Raise Levels of Attainment (UCORLA) in Bolton brought schools and practitioners together in eleven spotlight primary schools to plan a term's scheme of work

- Cultural Hubs in North Somerset connected schools with other young people's settings and agencies and cultural organisations and practitioners; each hub identified local needs and proposed projects, and most FYT project funding was routed through these hubs.

\footnotetext{
${ }^{9}$ Please note that given that none of the pathfinders were led by schools, and pathfinders treated schools as important among several partners, the evaluation did not pursue any additional lines of enquiry with schools in general or specialist schools in particular. The findings presented in this report reflect the perceptions of pathfinders and their partners and stakeholders in general.
} 
4.22 Pathfinders supported a wide range of school-based activities across a variety of cultural forms, ranging from the production of work at school to school trips to local cultural venues. The Telford pathfinder supported Maths Adventure, in which Year 9 students enthused Year 5 s about maths by creating a maths adventure trail through a local museum. The PUSH pathfinder supported Popey Art Skool, which aimed to raise awareness of local artists and galleries in Portsmouth at three primary schools, involving visits to city locations as well as school-based visits by local artists, to encourage children's artistic development. And Customs House created three hubs to deliver a sustained programme of after school activities for children.

4.23 Telford consultees indicated that participation in FYT had raised the expectations of teachers in terms of what children are capable of achieving and the quality of they subsequently cultural activities provided for them. There had been noticeable changes in teaching: staff had gained skills, had been inspired and were more confident when trying new approaches, and it was thought that their engagement with FYT would have a lasting legacy in their teaching practice.

\section{Consultation and engagement}

Involving children and young people

4.24 Pathfinders placed young people at the heart of the cultural offer in a variety of ways. They were active advisors and commissioners of work as well as participants in cultural activities. All pathfinders specified formal and informal methods for engaging young people in designing their offer, yet practical barriers meant that not all pathfinders managed to translate their aspirations into reality. Even so, by Year 2 explicit formal processes for engaging children and young people in design and delivery had been established by most pathfinders, and engagement mechanisms appeared to be having a positive impact on FYT delivery. The Create Culture Groups in North Somerset offered young people the chance to determine what was to be supplied in the area. In each group, young people were involved in the entire creative process, from undertaking research to liaising with cultural practitioners and managing budgets. The Youth Voice and Influence Programme was a key mechanism for the PUSH pathfinder to engage with young people in the development of its activities. It aimed to build relations with existing youth panels and fora, supporting and developing work with young people through a phased menu of creative programmes in order to inform future policy and design. In Leicester and Leicestershire, a key strand of the programme in Year 2 involved grants for different projects specifically commissioned by young people, alongside innovative approaches to engaging young people in decision making processes, with some acting as 'young dragons'. This process, modelled on the television programme Dragon's Den, provided young people with the opportunity to question organisations and providers applying for FYT funding about their proposal before deciding whether funding should be allocated. This process not only helped to engage young people but also provided a cross check on the demand for the activity amongst young people. Pathfinders had also adopted innovative ways of communicating the offer to young people; for example, a FYT facebook page started by Leicestershire, viral marketing campaigns by Leeds, and a new website for young people through the Telford Culture Zone. 
4.25 In targeting the hardest to reach, pathfinders sharpened their focus on commissioning projects for particular groups, specifically children under 5, young people who are NEET, LAC, and those with mental health issues, facing transport issues, or living in deprived areas. They targeted these groups in a number of different ways:

- $\quad$ Establishing links with specialist staff, and training practitioners

- $\quad$ Assessing and re-testing demand, and sharpening the focus on the target groups

- $\quad$ Engaging children and young people through formal and informal mechanisms

- $\quad$ Collecting ongoing feedback from projects and activities.

\section{Engaging parents}

4.26 Parents/carers' cultural participation has an influence over the participation of their children, so involving parents/carers and families is important in engaging particularly early years and primary-age children. The influence of peers grows stronger amongst older children and young people. Children's Centres and schools have an important role to play in encouraging parent/carers to engage in and extend the range and depth of cultural activities offered to their children. Parental opposition can prevent children from participating, so it is important to get them on side. Parents responding to the baseline survey also requested more information about the cultural offer in their area.

4.27 Pathfinders presented some good examples of engaging parents/carers and families in target groups for the cultural offer, including the following:

- $\quad$ Tower Hamlets - focused on fostering trust with BME parents via specific projects within a dedicated Family Engagement Strand of programme activities

- $\quad$ Customs House - kite- and banner-making workshops held at a Children's Centre over six weeks, involving disengaged parents/carers and also children and early years practitioners, resulted in better parent/child interactions at home, greater demand from parent/carers for Centre services, and Centre staff who were more confident in initiating cultural form activities

- $\quad$ Telford - teachers who have worked with disadvantaged primary school pupils reported that parents have raised their expectations of what their children can achieve and have seen the benefits of participating in cultural activities.

\section{Progression}

4.28 There were some examples of projects facilitating career progression and employment among young people. This did not feature strongly in most pathfinders, with the possible exception of Custom's House work with children who are at risk of becoming NEET in all secondary schools in the area. Whilst it is not clear why career progression and employment did not feature strongly in FYT, some pathfinders did succeed in identifying young people who were NEET and would benefit most FYT activity around progression, a illustrated below: 
- $\quad$ Liverpool - Pro-step, a project for young people who are NEET, resulted in positive progression into employment, education and training; of the nine participants, one entered full-time employment, one began a vocational placement, one had an interview for a work placement, and two pursued further education

- $\quad$ Shepway - Lydd Powerplay resulted in five of the more committed participants progressing from learning the basics of musical instruments and to writing their own music, including some who had never learnt a musical instrument before; some group members have formed a band which has performed in front of their school and generated a real sense of pride; some secondary school participants are intending to take music as an option for their GCSEs

- $\quad$ Customs House Creative Careers Events - these 'market place' events gave 350 secondary-school students and teachers the opportunity to gain insights into employment routes in the creative sector by talking directly to 45 practitioners and also agencies and organisations such as Connexions, Tyne and Wear Museums and local enterprise agency TEDCO

- $\quad$ Schools Choice Exhibition (PUSH) - this offered young people the opportunity to go 'behind the scenes' of Southampton City Art Gallery and, working with a visual artist, curate an exhibition of their own choice comprising their own collection and their responses to other works; the resulting exhibition remained open for a month and was visited by the Mayor.

\section{The reach and effectiveness of individual projects}

4.29 The evaluation is unable to comment on the extent and nature of reach of FYT funded projects across the ten pathfinder areas, mainly because at the time of writing this report, full monitoring data for Year 2 was not available. New activities appeared to form the bulk of all activities. Qualitative assessment by stakeholders interviewed during case study visits suggests that for the most part, projects set out in pathfinder delivery plans were delivered as planned, although Year 1 was slow to get started. They were generally confident that programme delivery was on track and had the potential to generate positive outcomes for participating children and young people. Their confidence was partly reliant on the fact that partnership and delivery structures were up and running, and key partners were on board.

4.30 Anecdotal evidence from the case studies suggests that participation may have been enhanced, especially amongst hard-to-reach groups. In some cases (PUSH for example), this was attributed by stakeholders to the fact that activities were led by young people. By combining the inputs of these young people with the experience and skills of practitioners and artists, the projects were able to reach significant numbers of young people, many of who had not participated in any prior activity.

4.31 Case study evidence and focus groups with young people show that the projects have been effective in other ways. The sheer range of projects on offer has provided young people with varied opportunities to test their interests, pursue their talents and raise their knowledge and understanding of what was on offer. Stakeholders and partners also cited some early benefits for young people participating in these projects, namely improved levels of confidence and 
self esteem. For one pathfinder (Customs House), a number of projects had been successful enough to be extended or repeated; these include a Creative Careers event and instrumental music workshops.

Table 4-1: Examples of effective projects

\section{Bolton Cell By Date Project}

This project was jointly funded by FYT and the North West Museums Hub, and 25 young people aged 16 and 17 from a local college took part. It was run by Bolton Museums with Genesis, an entry to employment agency. The project was aimed at getting to the hardest to reach and giving young people new employability skills. Although the project had an slow start, it picked up after a team building exercise, and the young people led the creation of an exhibition focused on crime and punishment. The exhibition received high praise locally and nationally. Participants were proud of their experience, and reported having gained confidence and benefiting from improved relationships with peers. Participants also gained project management and team working skills and spoke at several conferences.

\section{North Tyneside Children's Centre - Kite and Banner Project}

FYT coordinated a series of activities aimed at parents and young children within two Children's Centres in North Tyneside based in deprived areas. The areas are characterised by high deprivation, low cultural engagement and a lack of professional cultural activities. The project involved two artists working with a number of existing groups that meet at the centres. Over six weeks, the artists worked with parents and children aged 0-3 years to create kites and banners which were then used outside the two centres in permanent displays. The projects cost $£ 5,000$ for each centre. Although the number of participants was relatively small (27 adults and 35 children), participants were committed, given previous levels of engagement in the area. The projects involved CPD for practitioners at the centres, and gave parents creative ideas and empowered them to try these at home.

\section{Leeds Active Learning Literature Project}

Active Learning is a professional development programme created and run by Education Leeds Arts Forms, aimed at primary teachers and creative practitioners. Prior to FYT, Art Forms had run Active Learning Drama and Drawing modules. Following changes in the curriculum, there was a demand for arts skills development and greater understanding of how arts can be applied across the primary curriculum. The new module offered professional development learning for 12 primary school teachers and 12 art form specific practitioners, each course comprising four full training days. Teachers and practitioners were paired up to create partnerships. The sessions were developed by an experienced academic and professional storyteller who had never previously worked with the pathfinder.

Source: FYT pathfinder case studies.

\section{Quality}

4.32 Pathfinders recognised that delivering a high quality cultural offer involves much more than children and young people participating in high quality cultural activities, and involves the planning, design, delivery and evaluation of the cultural offer. Ensuring the quality of the cultural offer took a prominent position in pathfinder delivery plans, and was one of the key outcome measures for the evaluation. During Year 1, pathfinders developed systems and processes to measure and assess quality. In some areas there was also a strong drive to develop quantitative criteria or tools to provide comparable and consistent results. Leeds commissioned a specific piece of research assessing the national quality assurance frameworks currently in use and developing a badge system and code of conduct for creative practitioners.

4.33 Quality assurance mechanisms were often loosely defined, albeit present and acknowledged, and they included the following:

- In selecting providers, using those with demonstrable experience of working with children and young people and specific groups, of good repute and having sufficient safeguarding mechanisms (Leeds, North Somerset)

- $\quad$ CPD with delivery staff and practitioners (Liverpool, Tower Hamlets, Telford) 
- Quality assurance badge system and code of conduct for practitioners (Leeds)

- $\quad$ Use of the Arts Award accreditation scheme (PUSH)

- $\quad$ Integrating existing frameworks (Bolton)

- $\quad$ Taking young people's views into account in commissioning decisions (Leicestershire).

4.34 Some pathfinders devoted considerable energy to this issue. In Bolton, for example, a weeklong festival called 'That's Quality' gave young people the opportunity to explore what quality means to them. Partners were also encouraged to integrate existing quality frameworks such as Hear by Right and Quality in Study Support Framework (QiSS), and to use a quality kite mark aimed at giving parents an easy signal of the safety and quality of an activity.

4.35 Pathfinders were keen to consider both demand side and supply side definitions of quality, (e.g. the cultural experience for the participant young person, and the delivered cultural output from the provider). Several stakeholders were of the view that quality is subjective and defined entirely by the customer or end user, and should be based on a person's evaluation of their entire experience and not just its end product.

4.36 Case study evidence suggests that the programme did generate an improvement in the quality of the cultural offer in pathfinder areas. For example, consultees in Tower Hamlets were of the view that the pathfinder had made greatest progress in raising awareness of existing provision and increasing the coherence of the offer through the development of a strand-based programme of activity, all of which had CPD elements. In Bolton, growing membership of their Cultural Change teams and the increased levels of collaboration across and between partners suggest that the pathfinder's activities had generated a cultural change to improve quality of what was on offer.

4.37 In Telford, partners and providers consulted during the case study thought that the quality of provision was high and the programme had reached those who were furthest away from cultural experiences. PUSH stakeholders thought that their success lay in projects being influenced and led by young people. Leeds locality coordinators felt that as a result of the programme, there was stronger emphasis on sustained provision of cultural activities and a clear route for young people for pursuing opportunities.

4.38 It was clear from the case study consultations that quality was defined and measured by pathfinders using a multitude of factors, indicating that it is indeed multi-faceted. However, there is clearly a need for adopting a consistent framework that incorporates these factors and measures the success of the programme overall.

\section{Conclusion}

4.39 Over 200 interviews were conducted in 2009 and 2010 with pathfinder teams, partners, providers and other stakeholders in the ten pathfinder areas. Whilst the first year focused on understanding processes, structures and mechanisms that pathfinders were due to set up for delivering FYT, the second year attempted to understand the effectiveness of delivery. 
4.40 Stakeholders were very positive about the management and partnership arrangements for delivering the programme. These were beginning to generate positive benefits for the cultural infrastructure in pathfinder areas in the following ways:

- $\quad$ greater sharing and understanding of priorities for targeting young people and enhancing their cultural experiences

- $\quad$ appropriate protocols and networks to enable knowledge sharing and integration of activities among partners and providers

- $\quad$ commitment to sustainability by embedding FYT structures into existing priorities for children's services

- $\quad$ professional development and training for practitioners.

4.41 Partners played active and explicit roles in designing and commissioning of projects, and there were good examples of involving young people in leading and influencing the programme. New and innovative partnership working paved the way for better coordinated programmes of activity, and more informed audiences. Pathfinder leads and coordinators also played significant roles in driving forward FYT in their areas, actively seeking and maintaining partnerships and delivering effective projects.

4.42 No one model of delivery was most effective; rather, the ingredients for success lies in the extent to which the lead organisation was able to sustain contributions by its partners in delivering a multi-faceted programme involving a range of target groups, art forms and geographic areas. What also matters is provision that is demand led, well-coordinated and high-quality, judged both by supply-side professional excellence and the cultural experiences of children and young people. 


\section{5: Conclusion - making a difference}

\section{Introduction}

5.1 The central questions for any programme evaluation are: what difference has the programme made, and what changes has it brought about which would not have happened anyway? With $£ 25 \mathrm{~m}$ in planned programme funding distributed across ten pathfinder areas, these are not trivial questions. Before its termination, the evaluation had begun to collect evidence for answering these questions, with a particular focus on identifying 'what works' in providing a demand-led, local area-based and high-quality universal cultural offer for children and young people. These early results are discussed in this chapter.

\section{Impact}

5.2 The FYT programme represented a mix of top-down central government investment designed to catalyse innovative bottom-up delivery solutions in response to local needs. Hence it was important for the evaluation to identify what difference the programme had made to children and young people at an aggregate, all-programme level, but also to observe new approaches to local planning and delivery that are important in bringing about this change. The findings from Year 1 and 2 demonstrate early evidence of programme additionality in several areas, as observed and reported by partners and stakeholders:

- An enhanced cultural offer for young people that is of high-quality and better coordinated

- Improved communication and awareness among children and young people about what is on offer

- $\quad$ Reaching out to children and young people in school and out-of-school settings in new areas and for new participants

- $\quad$ Networking and brokerage among partners, providers and practitioners, developing a shared understanding of what delivering an demand-led, local area-based, highquality cultural offer means.

5.3 The programme appeared to have gained strategic added value ${ }^{10}$, as demonstrated by the partnership structures and processes that pathfinders have set up as part of the FYT delivery infrastructure, and providing strategic leadership (evident in management and governance arrangements) using the FYT investment. Case study consultees were of the view that joinedup working had taken place, and as pathfinders continued in their capacity building efforts, delivery partners and providers were beginning to collaborate effectively. Investment in the

\footnotetext{
${ }^{10}$ Strategic added value is a measure of the impact of strategic leadership, partnership creation and decisionmaking. It is the added value that is expected to be realised by co-ordinating the outputs from a programme and influencing others to help achieve programme outputs and outcomes. Although the concept was devised for Regional Development Agency impact evaluations, it is also applicable to other strategic bodies that can influence their partners' and stakeholders' behaviour.
} 
strategic development of cultural providers was seen by one pathfinder as a key area of additionality.

5.4 In the absence of follow-up post-programme survey data it is difficult to quantify the impact of the programme on outputs and outcomes for children and young people. Nevertheless, the largely qualitative evidence from over 28 focus groups with children and young people who had attended FYT activities indicates some early signs of the ways in which programme activities had benefited them, including increased aspirations and confidence and progression into education or employment:

- $\quad$ Children and young people found FYT activities enjoyable and engaging

- $\quad$ They reported that these offered something new and more focused for them

- $\quad$ They produced creative outputs that involved effective team working

- $\quad$ There was evidence of improved confidence among participants

- $\quad$ There were also examples of practical skills and knowledge development

- $\quad$ Participants were keen to come back for more.

5.5 Pathfinders cited anecdotal evidence that participation in cultural activities had increased as a result of FYT. One pathfinder (Leicestershire) noted that 30,000 young people had engaged in their 'Go and See' visits, and there was some evidence that a good number of participants had sustained involvement over a longer period of time.

5.6 The case studies also provided evidence of greater parental engagement with cultural activities. Parent/carers' cultural participation influences the participation of their children, so involving parents/carers and families is important for engaging particularly early years and primary-age children (the influence of peers grows stronger amongst older children and young people). Children's Centres and schools have an important role to play in encouraging parents/carers to engage in and extend the range and depth of cultural activities offered to their children. Conversely, parental opposition can prevent children from participating, so it is important to get them on side and, as the baseline survey data suggest, providing more information about the cultural offer in local areas would help to achieve this. Case studies highlighted a number of useful examples of engagement with parents:

- $\quad$ Tower Hamlets Family Engagement Programme - established as one of the key strands of the programme, it involved production of a 'family friendly' venues guide outlining venues which have regular affordable (either free or low cost) family provision, and devolved budgets to Local Area Partnership (LAP) coordinators to encourage/support families who wouldn't usually engage to take up the available offer ( $£ 5000$ per LAP per term)

- $\quad$ Telford - teachers working with disadvantaged primary school pupils reported that parents have raised their expectations of what their children can achieve and have experienced the benefits of cultural activities themselves. 


\section{Sustainability}

5.7 The issue of sustainability clearly took centre stage for FYT pathfinders when it came to setting up management and governance structures and mechanisms for delivery of the programme. As discussed in earlier sections, the approaches adopted involved embedding the cultural offer within existing structures and the local context, and leaving a legacy.

5.8 Furthermore, many pathfinders demonstrated their commitment to sustainability and indicated the ability of elements of the programme to sustain itself beyond its funding life, by investing in CPD and setting up networking protocols that partners could continue to use and benefit from. Some stakeholders identified culture change and shared understanding as a key plank of achieved sustainability. The goal was to create an infrastructure that continues to generate opportunities for young people beyond the lifetime of FYT funding, and which did not necessarily require significant additional investment. North Somerset, for example, created a series of 'legacy' projects that were designed to attract organisations, especially schools, to continue investing in cultural activities beyond FYT.

5.9 Several pathfinders were successful in attracting significant additional investment from partners as a way of ensuring sustainability for FYT project activities. By December 2009, projects in Telford had secured approximately $£ 389,000$ in (planned) match funding from a FYT (planned) budget of $£ 586,000$. Existing projects secured a greater share of this match funding (60\%, compared with $40 \%$ for new projects $40 \%)$, suggesting that the pathfinder's efforts to mainstream project activities were working. Leveraged funding sourced from a variety of organisations and individuals, including schools, the then Learning and Skills Council, the MLA, Arts Development, Birmingham REP, Ideas Foundation and participant registrations. Bolton took the innovative step of creating a Funding and Partnerships Officer post whose role was to work with core FYT partners to identify and apply for alternative sources of funding to build capacity. In addition, PUSH secured over $£ 500,000$ in matched funding for FYT proejcts, and the Leeds pathfinder established the principle of part-funding for some of their FYT activities in order to ensure greater commitment and ownership from delivery partners.

\section{Messages for local authorities}

5.10 The evaluation findings to date enable several conclusions to be drawn for developing a local area-based, demand-led and high-quality cultural offer for children and young people. The findings allow for implications to be identified for future provision in an austere financial environment where cost-effective provision will become ever more important.

\section{Delivery models}

5.11 The sharpening of focus by pathfinders in adopting a 'progressive universalism' approach towards vulnerable groups and areas indicates a continuing need for targeting provision for groups and areas where significant returns are likely to be gained. This is rightly so, given that participation generally is high and there are specific sub-groups (secondary-aged children, boys, ethnic minorities, poor communities) that are the low participators. 
5.12 In the absence of continuing central government funding and given the paucity of information about existing cultural activities reported by parents in some areas, a stronger emphasis should be placed on improving the coordination, coherence and communication of the existing cultural offer and extending its reach to ensure wider participation. Pathfinders had successfully set several activities in train to achieve this - establishing networks of providers and partners, CPD for practitioners and taster sessions for children and young people, and listening to what sorts of cultural activities they wanted for their local areas. Communicating the offer is very important, and new approaches are needed to encourage those who think that engaging in a wide range of different cultural experiences is 'not for me'.

\section{Delivery and partnership structures}

5.13 In the current financial and political environment, reduction and consolidation of the wide range of agencies which currently support local cultural activities is likely, and this may lead to a simplification of local area structures for delivering a future universal cultural offer. The FYT pathfinders engaged multiple (often cultural form-specific) agencies as strategic partners and leveraged additional funding from them in order to extend the work of FYT delivery partners. Reduction and consolidation of agencies would thus lead to simplified partnership arrangements and also fewer sources of additional funding. However, encouraging children, young people and families who are 'low participators' to discover a wider range of cultural experiences is a necessarily localised and complex process, involving close working with early years settings and schools, cultural organisations and practitioners, and community and voluntary sector (VCS) organisations.

5.14 Developing partnerships between local public and VCS organisations will undoubtedly become more important, and the pathfinders display good practice in this area. They have set up local area groups comprising delivery partners and sometimes also children and young people. With strategic direction typically provided by a central coordinating team and an advisory group, these local 'engagement groups' have been responsible identifying needs, developing elements of the offer and project ideas, and helping to select high-quality provision. This level of community engagement is likely to remain a necessity for any future delivery of a universal cultural offer.

5.15 For a local area-based, demand-led and high-quality universal cultural offer to succeed, areabased coordination is required. Pathfinder experience suggests that local authorities can play effective roles in determining need, planning provision, coordinating delivery, commissioning supply, and assuring quality. Strong local authority involvement - either as pathfinder lead organisations or working alongside lead cultural organisations - affords opportunities to link the cultural offer to other area-based priorities for children, families and communities. Several pathfinders connected the development of a universal cultural offer to wider policies, strategies and initiatives for children and young people in order to secure the future relevance and wider support for investment in the cultural offer.

5.16 Pathfinder experience also shows that local authorities can play important roles in brokering partnerships between Children's Centres, schools and other children's venues seeking to access local, regional and national cultural organisations and networks of practitioners. Pathfinder experience suggests that even with devolved budgets, schools will still need to find 
good cultural organisations and practitioners to work with, and also other schools with good practice to share on how cultural experience enhances children's learning. It is likely therefore that the brokerage services created by some pathfinders will still be in demand.

\section{Engaging schools}

5.17 Pathfinders involved schools in the design and delivery of the cultural offer to children and young people in a variety of ways, most typically as delivery partners or representatives on advisory groups rather than as strategic partners. The central place schools occupy in their communities was recognised as an opportunity to engage large numbers of children and young people in creative learning and cultural form practice linked to curriculum delivery and also out-of-school activities. The baseline survey findings also reveal that parents/carers want schools to play a greater role in telling them about the cultural opportunities which are available in their areas.

5.18 Despite the central roles schools played in the design, delivery and communication of the cultural offer in some pathfinder areas, several pathfinders struggled to engage schools and secure their participation. There were several reasons for this. It was reported that specialist arts colleges did not think that FYT added significantly to what they already offer their pupils, and pathfinders had difficulty persuading them to share their resources with other schools and their communities. Other schools were sometimes deterred by FYT application processes for securing project funding, or felt unable to prioritise FYT over more pressing priorities for their pupils, or did not have the capacity to engage with yet another external initiative. The lack of connectivity between FYT and curriculum delivery was also presented as an obstacle.

5.19 Schools and colleges that did engage with FYT reported positive results, particularly when cultural practitioners worked alongside teachers and developed their confidence and capacity for supporting creative learning and developing cultural form practice with their pupils. Giving young people the opportunity to meet people who work in the creative and cultural industries opened their eyes to the wide range of employment prospects the sector offers, and made them aware of education and training pathways. Schools also worked with community and cultural organisations to identify the sorts of cultural experiences that would appeal to their pupils, brought large numbers of children and young people into cultural venues they would otherwise not have visited, and provided arts-based after-school activities.

5.20 Involving schools in the design, delivery and communication of a local area-based, demandlead and high-quality cultural offer for children and young people thus remains a priority for local authorities and cultural organisations. Another challenging priority going forward is enabling children and young people and their families to become more actively involved in the design and delivery of the cultural offer, not just as participants and creators in different cultural forms, but also as advisors, innovators, promoters and commissioners of local cultural projects and activities. More active participation in creating the cultural offer will strengthen links to other services for children and young people and create opportunities for embedding cultural activities across a wide range of related policies and strategies. Doing so will help local authorities and their partners to make the case for continuing investment in cultural provision targeting 'low participators' within a progressively universal offer for all. 
Ref: DFE-RR089

ISBN: 978-1-84775-870-5

(C) Department for Culture, Media and Sport and the Department for Education March 2011 